\title{
An Analysis of Dielectric Parameters and Penetration Depth of Tomato Sauces
}

Satyendra Pratap Singh*

Department of Physics, AIAS, Amity University, Noida-201301, India

\begin{abstract}
The dielectric constant and dielectric loss of the Tomato Sauce have been measured at different moisture levels in the radio frequency range i.e. $100 \mathrm{kHz}$ to $1 \mathrm{MHz}$. The measurements were carried out in the temperature $25^{\circ} \mathrm{C}$ to $50^{\circ} \mathrm{C}$. The dielectric properties show remarkable changes as the moisture levels i.e. salt content vary. The dielectric properties have also been used to determine the penetration depth. The penetration depth was found to be quite high in radio frequency range in comparison to penetration depth for microwaves.
\end{abstract}

Keywords: Dielectric; Tomato sauce; Moisture levels; Penetration depth

\section{Introduction}

The dielectric properties of materials are important because these properties indicate the interaction of electromagnetic energy with the material at microwave frequency as well as radio frequency. The dielectric properties have been defined and discussed in detail by Nelson [1]. The major interest in microwave dielectric properties of agriculture products are for their use in microwave and radio frequency dielectric heating.

The microwave and radio frequency dielectric heating of agriculture and food materials are important because they influence the way in which electromagnetic field associated with electromagnetic energy interact with the materials. Dielectric data developed by Von Hipple [2] for a variety of organic and inorganic material over a broad range of frequencies and temperatures from the basis for commercial dielectric heating system. Kent [3] presents a comprehensive review of available dielectric data for food and other biological material.

Over the past 60 years, numerous studies have been done on microwave heating. An advantage of microwave heating over the conventional thermal processing is the rapid heating by direct interaction between microwave and foods that are hermetically sealed in microwaveable packages [4]. The microwave pasteurization and sterilization can potentially improve the product quality [5]. But microwave heating is limited to small sized food packages due to relatively small penetration depth of microwaves in food materials. This limitation can be overcome by using radio frequency heating.

The principles of radio frequency heating are very similar to microwave heating. Heat is generated within dielectric materials such as foods, when the electromagnetic field reverses the polarization of individual molecules or cause migration of ions within the material as it alternates at high frequency. The main difference between microwave and radio frequency is the wavelength. Radio frequency heating may be particularly useful when applied to institutional sizes packaged food products in big boxes because of deep penetration.

Cathcart and Park [6] first studied the use of radio frequency heating to thaw frozen eggs, fruits, vegetables and fish. Radio frequency dielectric heating is now widely used in industrial applications such as drying textile products (spools, rovings, and skins) final drying of paper, final dehydration of biscuits at outlets of baking ovens and melting honey. However possible non-uniform temperatures within products and difficulty in the application of overpressure during radio frequency heating have previously stood as major barriers to developing applications of radio frequency in sterilization and cooking food stuffs.

Dielectric properties of various food materials have been reported in the literatures $[7,8]$. Most research on the dielectric properties of food has been carried out on solid foods. For liquid foods, microwave heating rates have been reported $[9,10]$.

In the present paper, we have investigated dielectric properties of Tomato sauce using radio frequency signal. The ultimate objective of this study is to develop Low Temperature Short Time (LT ST) sterilization protocols for foodstuffs using radio frequency heating.

\section{Materials and Method}

\section{Materials}

Tomato sauce was purchased by certified Agro Foods NIJJER limited Company at Amritsar. Tomato sauce is made by Tomato paste, sugar, salt, acetic acid, thickener, onion powder, garlic powder, mixed spice.

\section{Method}

The capacitances $\left(\mathrm{C}_{\mathrm{p}}\right)$ and dissipation factor $\left(\mathrm{D}_{\mathrm{p}}\right)$ measurements have been made with the help of Impedance/Gain Phase Analyzer (Model No.-HP-4194A, range of frequency $100 \mathrm{~Hz}$ to $40 \mathrm{MHz}$ ) using specially designed open ended coaxial probe for permittivity measurement of agricultural product. The sample holder has been gold plated to reduce dissipation losses. It is calibrated by using standard liquids (Benzene and Methanol) and the error in measurement for $\epsilon^{\prime}$ was found to be less than $\pm 1.0 \%$ and for $\epsilon^{\prime \prime}$ was $\pm 1.5 \%$. The dielectric parameters have been calculated with the help of the following relations

$$
\begin{gathered}
\varepsilon^{\prime}=\frac{C_{P}-C_{0}}{C_{G}}+1 \\
\tan \delta=\frac{C_{P} D_{P}-C_{O} D_{O}}{C_{P}-C_{O}}
\end{gathered}
$$

*Corresponding author: Satyendra Pratap Singh, Department of Physics, AIAS, Amity University, Sector 125 Noida-201301, India, Tel: +919953776787; +917905651988; E-mail: spsingh9@amity.edu

Received April 25, 2018; Accepted July 17, 2018; Published July 20, 2018

Citation: Singh SP (2018) An Analysis of Dielectric Parameters and Penetration Depth of Tomato Sauces. J Food Process Technol 9: 742. doi: 10.4172/2157 7110.1000742

Copyright: () 2018 Singh SP. This is an open-access article distributed under the terms of the Creative Commons Attribution License, which permits unrestricted use, distribution, and reproduction in any medium, provided the original author and source are credited. 


$$
\tan \delta=\frac{\varepsilon^{\prime \prime}}{\varepsilon^{\prime}}
$$

Where $\mathrm{C}_{\mathrm{p}}$ is the Capacitance of Sample holder with sample, $\mathrm{C}_{\mathrm{o}}$ is the Capacitance of empty sample holder, $\mathrm{C}_{\mathrm{g}}$ is the geometrical capacitance $\left(\mathrm{C}_{\mathrm{g}}=\mathrm{q} / \mathrm{v}=2 \pi \varepsilon_{0} \mathrm{~h} / \log _{\mathrm{e}}(\mathrm{b} / \mathrm{a})=1.46\right.$ where $\mathrm{a}$ and $\mathrm{b}$ are the internal and external radii respectively), $\varepsilon_{0}$ is the permittivity of free space, while $\omega$ represents angular frequency.

\section{Moisture content}

Moisture contents in both the sauce were determined on a wet basis. Four different concentrations of Tomato sauce were used. Samples were prepared by adding distill water in tomato sauce at mixture ratio of $0.00: 1.00,0.33: 1.00,0.50: 1.00,1.00: 1.00$ (water: sauce). These mixtures were stored in sealed jars at $15^{\circ} \mathrm{C}$ and permitted to reach room temperature $\left(24^{\circ} \mathrm{C}\right)$ in sealed jars before opening for measurements. The samples were kept in this condition for about 48 hours before the measurements were made. Moisture content of the prepared samples has been determined as $73 \%$ and $69 \%$ by approved oven method [11].

\section{Penetration depth}

Frequency of the wave is also an important factor and the power absorption by food material also depends on the square of the electric field intensity.

For a plane wave the electric field intensity $E$, which has $\mathrm{e}^{\mathrm{j} \omega t}$ dependence can be given by [2].

$$
\mathrm{E}(\mathrm{z})=\mathrm{E}_{0} \mathrm{e}^{\mathrm{j} \omega t}-\gamma^{z}
$$

Where $E_{0}$ is root mean square electric field intensity at the point of reference, $\mathrm{t}$ is time, $\gamma$ is propagation constant and it is defined as

$$
\gamma=\alpha+j \beta=j\left(\frac{2 \pi}{\lambda}\right) \sqrt{\varepsilon}
$$

Where $\alpha$ attenuation is constant, $\beta$ is phase constant, and $\lambda$ is wavelength. The attenuation constant can be calculated by

$$
\alpha=\omega \sqrt{\mu \varepsilon_{0} \varepsilon^{\prime}}\left[\left(\frac{\left(\left\{1+\left(\frac{\varepsilon^{\prime \prime}}{\varepsilon^{\prime}}\right)^{2}\right\}\right)^{1 / 2}-1}{2}\right)\right]^{1 / 2}
$$

The dielectric properties of materials are very important in evaluating the penetration of energy can be achieved. Penetration depth, D, is defined as the depth where the Power drops to 1/e of its surface value. It can be calculated using following equation

$$
D=\frac{1}{2 \alpha}
$$

\section{Result and Discussion}

The dielectric properties of liquid foods vary with several different factors. In hygroscopic materials, the amount of water in the materials is generally a dominant factor on dielectric properties. The dielectric properties also depend upon frequency of applied field, temperature and density of the materials. Of course, the dielectric properties of liquid materials are also dependent on their chemical composition an especially on the permanent dipole moments associated with water.

\section{Frequency dependence}

Dielectric properties for $100 \mathrm{kHz}$ to $1 \mathrm{MHz}$ has been considered in more details, because these frequencies have been internationally approved for radio frequency heating. The dielectric properties of most materials vary considerably and decrease with the frequency of applied field with the expectation of low loss materials i.e. the materials that absorb essentially less energy from radio frequency. This frequency dependence has been discussed previously by Nelson and other [1].

The value of dielectric constant and dielectric loss is decreasing almost linearly with frequency in temperature range $25^{\circ} \mathrm{C}$ to $50^{\circ} \mathrm{C}$ for Tomato sauce.

In Figures 1 and 2, dielectric constant and dielectric loss were plotted against frequency. Dielectric constant decrease steadily for all given concentration of water while dielectric loss decreases more rapidly for pure samples i.e. the insignificant effect of salt on variation of

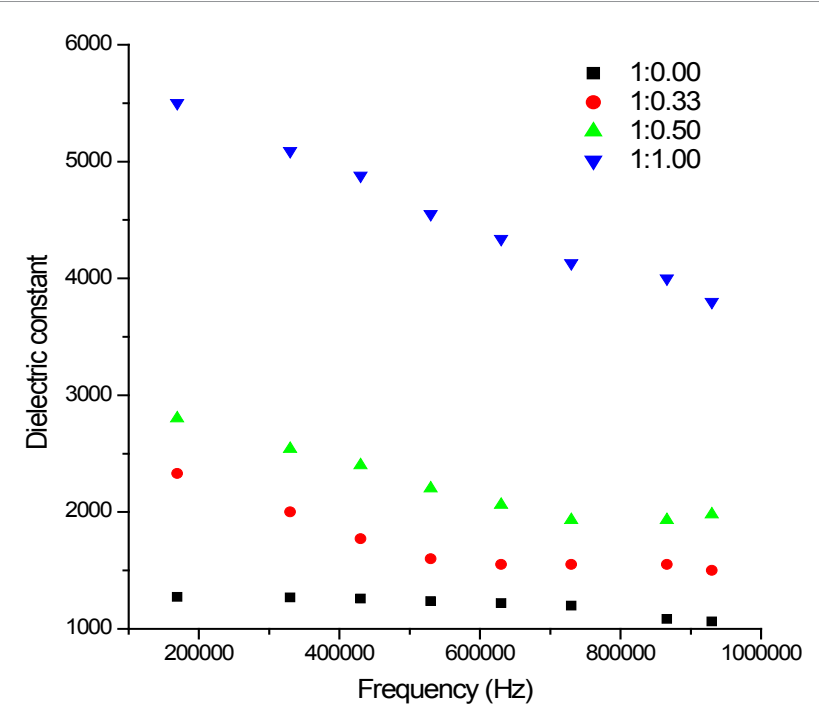

Figure 1: Dielectric constant verses frequency indicated moisture content and at constant temperature $\left(45^{\circ} \mathrm{C}\right)$.

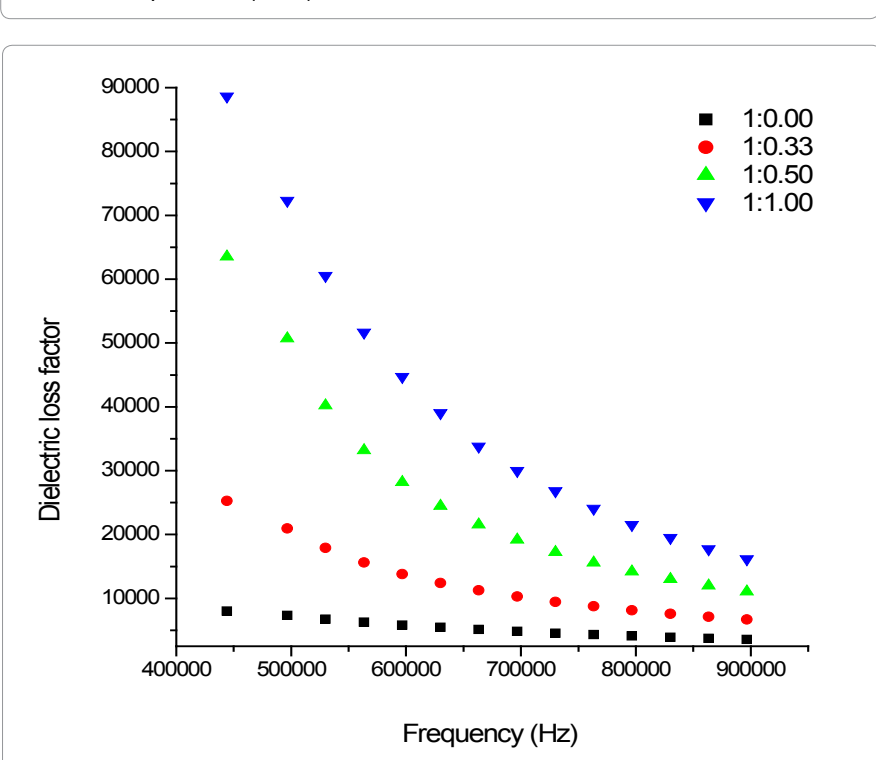

Figure 2: Dielectric loss factor verses frequency indicated moisture content and at constant temperature $\left(45^{\circ} \mathrm{C}\right)$. 
$\varepsilon^{\prime}$ with frequency is consistent with data presented by other researchers for various food products [3]. Dielectric constant is less dependent on salt content while dielectric loss decreases significantly sample having less salt [7]. This behavior is also apparent in the present work.

\section{Moisture dependence}

It is clear from Figures 3 and 4 , that for a given frequency and temperature, $\varepsilon^{\prime}$ and $\varepsilon^{\prime \prime}$ of the complex dielectric permittivity increases with increase in the moisture levels for all given temperatures and frequencies.

The rate of increase of $\varepsilon^{\prime}$ and " with the moisture content is high at low frequencies. This is obvious from the fact that at high moisture level more water dipoles contribute to the polarization, due to high water mobility, showing that the water dipoles easily follow the applied field variations. At low moisture contents, particularly below $0.4 \%$ both $\varepsilon^{\prime}$

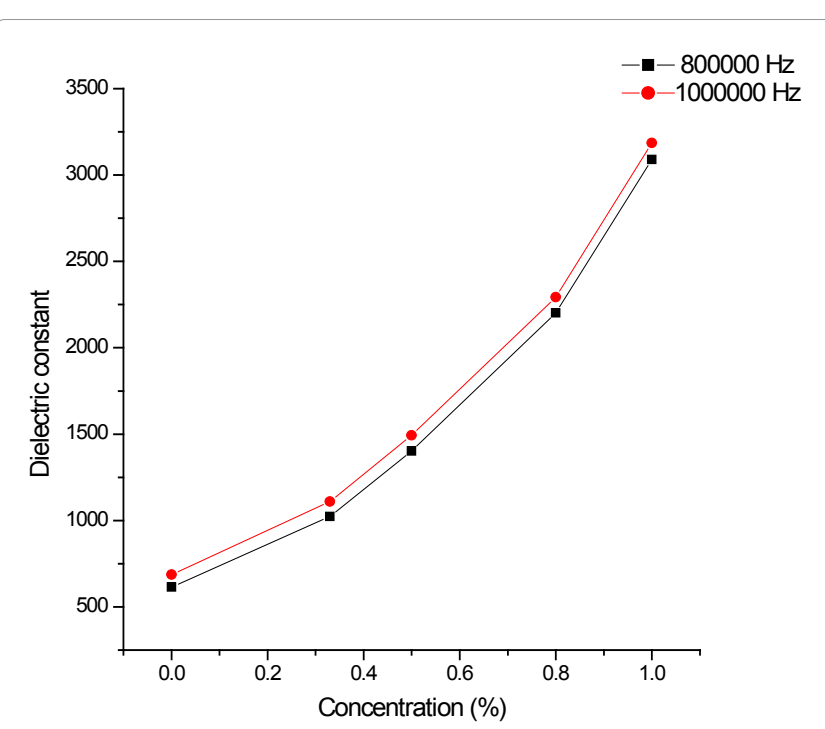

Figure 3: Dielectric constant verses moisture content at indicated frequency and at constant temperature $\left(45^{\circ} \mathrm{C}\right)$

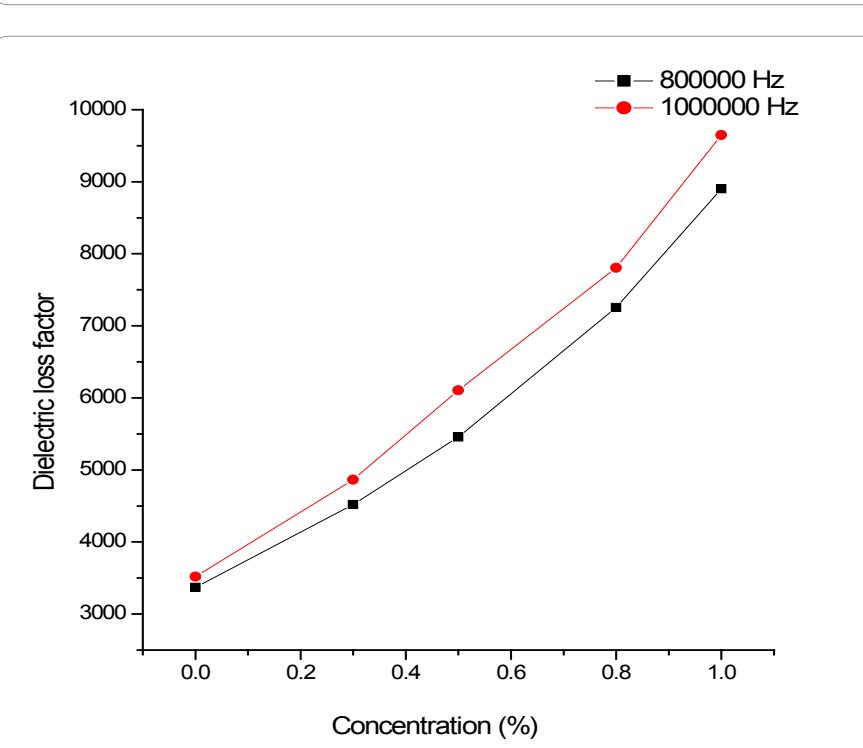

Figure 4: Dielectric loss factor verses moisture content at indicated frequency and at constant temperature $\left(45^{\circ} \mathrm{C}\right)$ and $\varepsilon^{\prime \prime}$ of the complex permittivity are small. This is because of strong bound water state (monolayer) where distance between the water molecule and cell wall is very small and attraction force is very large. Therefore, the dielectric constant and dielectric loss both are small.

As moisture level increases beyond $0.4 \%$ increase in the both the, $\varepsilon^{\prime}$ and $\varepsilon^{\prime \prime}$ of the complex permittivity accelerates and this trend could be attributed to change of bound water state from first (monolayer) to second (multilayer) type. Sharp increase is noticed for all the frequencies for the moisture content reaching beyond $0.8 \%$. This behavior could be attributed to the transition of bound water state second (multilayer) to third (osmotic tension) type or Free State water. At high moisture level and low frequency, the ionic conductivity is high, therefore for such moisture level and frequencies, the dielectric losses are considerably high.

\section{Penetration depth}

It is an important parameter in characterizing temperature distribution in radio frequency heated materials.

In general, the large penetration depth, the more uniform the temperature distributions would be for treatment. But in earlier study microwave heating is used for other sauce and sake [12], which is limited to small sized food packages due to relatively small penetration depth of microwaves.

Figure 5 shows that the variation of penetration depth with frequency. It is clear from the graph; the value of penetration depth is quite high as compared to penetration depth form microwaves. The value of penetration depth decreases continuously with increase in frequency for observed set of frequency from $100 \mathrm{kHz}$ to $1 \mathrm{MHz}$ at fixed temperature $45^{\circ} \mathrm{C}$.

Figure 6 shows the temperature dependence of penetration depth. The value of penetration depth decreases significantly with increase in temperature because of ionic and dielectric losses interaction. However, this behavior can be explained theoretically using Cole-Cole relation with addition of loss due to ionic conduction. ‘

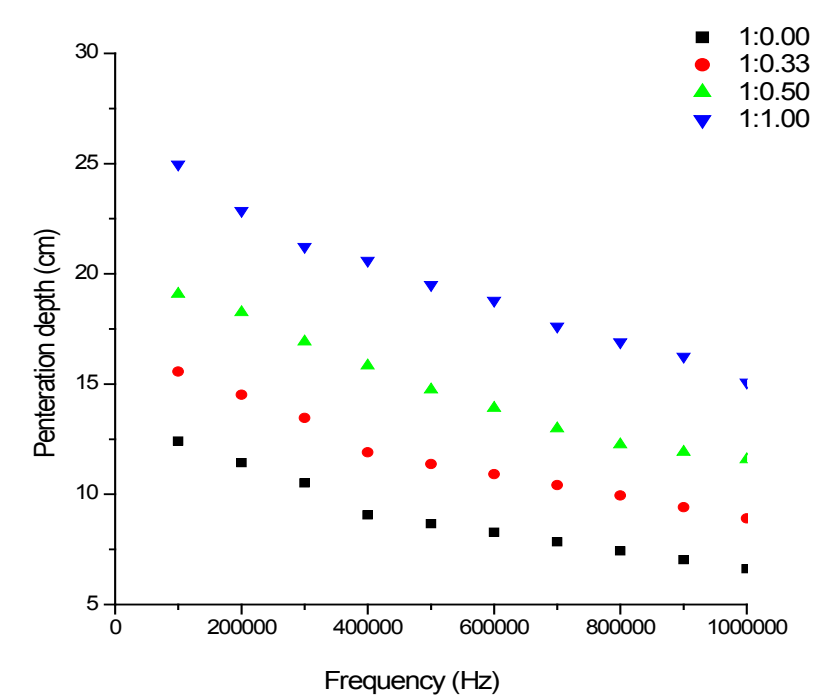

Figure 5: Penetration depth verses frequency at indicated moisture content and at constant temperature $\left(45^{\circ} \mathrm{C}\right)$. 
Citation: Singh SP (2018) An Analysis of Dielectric Parameters and Penetration Depth of Tomato Sauces. J Food Process Technol 9: 742. doi:

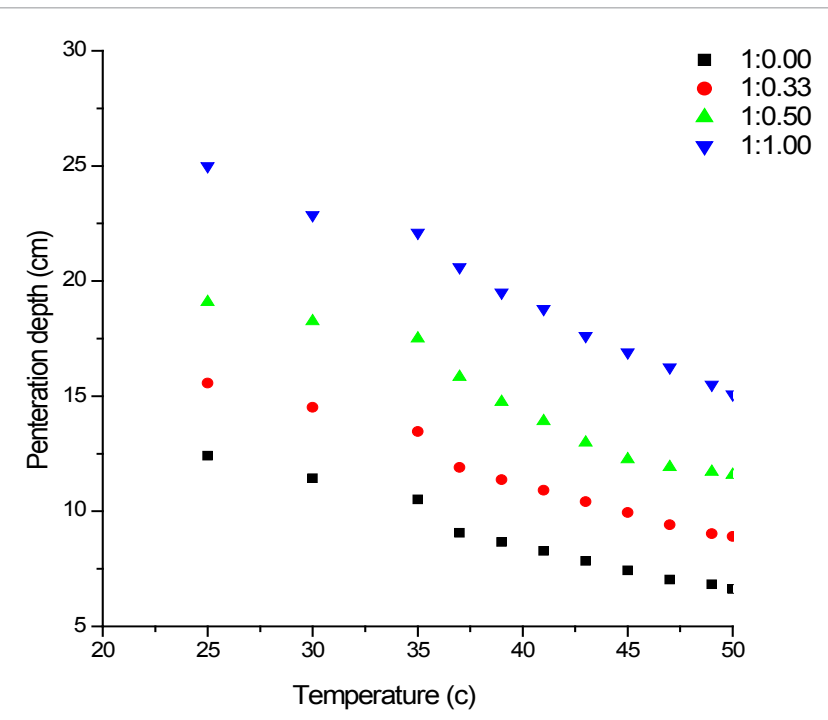

Figure 6: Penetration depth verses temperature at indicated moisture content and at frequency $1 \mathrm{MHz}$.

\section{Conclusion}

1. The results of the present work clearly indicate that the dielectric constant and dielectric loss are dependent on frequency, temperature and moisture levels of the samples. The dielectric constant can be used as a measure of salt and water content of the food stuffs.

2. The higher penetration depth for radio frequency in comparison to microwaves clearly shows use of radio frequency signal for sterilization of foodstuffs by radio frequency dielectric heating.

3. The penetration depth values have been analyzed with the variation of frequency and temperature. It is found that the penetration depth decreases with increase in temperature.

\section{References}

1. Nelson SO (1973) Electrical properties of agricultural products-A critical review Trans ASAE 16: 384-400.

2. Hipple V (1954) Dielectric materials and applications. MIT Press, Boston, MA.

3. Kent M (1987) Electrical and Dielectric Properties of Food Materials. Sci Technol.

4. Harlfinger L (1992) Microwave sterilization. Food Technol 46: 57-60.

5. Ohlsson T (1989) Dielectric properties and microwave processing. Food Properties and Computer-Aided Engineering of Food Processing Systems 168: 73-92.

6. Cathcart WH (1946) Defrosting frozen foods by high frequency heat. Food Res 11: $341-346$.

7. Goedeken DL (1997) Dielectric Properties of a Pregelatinized Bread System at $2450 \mathrm{MHz}$ as a Function of Temperature, Moisture, Salt and Specific Volume. J Food Sci 62: 145-149.

8. Nelson SO (1994) Measurement of microwave dielectric properties of material J Food Eng 21: 365-384.

9. Singh SP, Chandel VS, Manohar R (2018) Dielectric study of clove oil. J Ayurveda Integr Med. 9: 53-56.

10. Shibukawa S, Shokuhin Kanetsu S (2005) Dielectric properties of soya sauce Asakura Shoten Tokyo 71: 92-97.

11. USDA (1971) Oven methods 6: 916-926.

12. Tanaka F, Morita K (2002) Analysis of dielectric properties of rice vinegar and sake. Trans ASAE 45: 733-740. 